\title{
Dust-acoustic solitary structures in plasmas with nonthermal electrons and positive dust
}

\author{
F. Verheest ${ }^{1,2}$ and S. R. Pillay ${ }^{2}$ \\ ${ }^{1}$ Sterrenkundig Observatorium, Universiteit Gent, Krijgslaan 281, B-9000 Gent, Belgium \\ ${ }^{2}$ School of Physics, University of KwaZulu-Natal, Private Bag X54001, Durban 4000, South Africa \\ Received: 4 April 2008 - Revised: 9 June 2008 - Accepted: 17 June 2008 - Published: 14 July 2008
}

\begin{abstract}
Large dust-acoustic solitons and kinks in dusty plasmas with positive cold dust, nonthermally distributed electrons and Boltzmann ions have been studied in a systematic way, to delimit their compositional parameter space. The existence domain of positive solitons is limited by infinite dust compression, of negative ones by the occurrence of potential kinks, provided the electrons are sufficiently nonthermal and there is sufficient positive charge on the dust. There is a parameter range where both negative and positive solitary structures coexist.
\end{abstract}

\section{Introduction and basic formalism}

Dusty plasma physics studies the properties of heavier charged dust in the presence of traditional electrons and ions. These mixtures occur in heliospheric and astrophysical plasmas, and in laboratory and technological applications (Verheest, 2000; Shukla and Mamun, 2002). Many treatments describe the dust as negative ions, in view of the electron current charging models, but in other environments one may encounter positively charged dust, e.g. due to photoelectric effects. There is a recent interest in how waves are modified by this change of dust charge sign and by the concomitant excess of electrons over protons (Sakanaka and Shukla, 2000; Mamun and Shukla, 2002; Baluku et al., 2008; Mamun, 2008).

At the lowest frequencies the dust motion has to be taken into account, leading to dust-acoustic modes (Rao et al., 1990; Verheest, 1992; Barkan et al., 1995). On the other hand, space plasma observations indicate the presence of ion and electron populations which are not in thermodynamic equilibrium (Asbridge et al., 1968; Feldman et al., 1983;

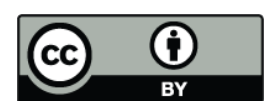

Correspondence to: F. Verheest

(frank.verheest@ugent.be)
Lundin et al., 1989; Futaana et al., 2003). Often, these nonthermal velocity distributions include a ring structure, and the simplest analytical way to model such effects is by the Cairns distribution (Cairns et al., 1995). Combining these different strands, we study dust-acoustic solitary structures in plasma containing nonthermal electrons, hot positive ions and cold, positive dust grains, with respective subscripts $e, i$ and $d$.

We follow recent treatments of nonthermal effects on dustacoustic solitons (Mendoza-Briceño et al., 2000; Maharaj et al., 2006; Verheest and Pillay, 2008), suitably modified for the presence of positive dust. Since the algebra runs along analogous lines as in our recent paper (Verheest and Pillay, 2008), we can be brief and present only the highlights. Although the figures in the present paper look identical to our earlier ones (Verheest and Pillay, 2008), they are generated in a quite different normalization and, more importantly, have a different interpretation.

The fraction of positive charge residing on the dust is $f=Z_{d} n_{d 0} / n_{e 0}$ and on the ions $1-f=n_{i 0} / n_{e 0}$, where $n_{d 0}$, $n_{e 0}$ and $n_{i 0}$ represent the number density of the positive dust, positive Boltzmann ions and nonthermal electrons, respectively, and $Z_{d}$ is the number of (positive) unit charges on the charged dust grains.

In a Cairns distribution (Cairns et al., 1995) the (normalized) electron density is given by

$n_{e}=\frac{1+3 \alpha-4 \alpha \varphi+4 \alpha \varphi^{2}}{1+3 \alpha} \exp [\varphi]$.

Here the electrostatic potential $\varphi$ has been normalized to $\kappa T_{e} / e, T_{e}$ being the temperature the electrons would have in the absence of nonthermal effects, and $n_{e}$ has been derived by integration of a phase space Cairns distribution function, expressed in terms of a parameter $\alpha$ characterizing the degree of nonthermality (Cairns et al., 1995). Such distributions are the simplest ones to model superthermal wings. We will use

Published by Copernicus Publications on behalf of the European Geosciences Union and the American Geophysical Union. 
values up to 0.6 to detect how $\alpha$ influences the existence domains for solitary structures.

The mobile ions have been assumed Boltzmann distributed, with

$n_{i}=(1-f) \exp [-\tau \varphi]$,

where $\tau=T_{e} / T_{i}$ is the electron-to-ion temperature ratio. The cold dust is described by the standard continuity and momentum equations (Verheest and Pillay, 2008), but now referred to a different normalization with i.a. a dust-acoustic speed $C_{d a}=\left(Z_{d} \kappa T_{e} / m_{d}\right)^{1 / 2}$. We want to focus on the influence of $f$ and $\alpha$, and it is more transparent to keep those parameters outside the normalization.

In the model under consideration, both the Boltzmann ions and the nonthermal electrons are subsonic in a fluiddynamical picture (McKenzie, 2002; Verheest et al., 2004). Subsonic (hot) and supersonic (cool) are defined by comparing the nonlinear structure velocity $U$ to each species' thermal velocity, and for the existence of solitary structures we need at least one subsonic and one supersonic species (McKenzie, 2002; Verheest et al., 2004). In a frame where the nonlinear structure is stationary $(\partial / \partial t=0)$ and all variables tend to their undisturbed values at $x \rightarrow-\infty$, in particular $\varphi \rightarrow 0$ and $u_{d} \rightarrow M$, we can integrate the dust equations with respect to $x$ and find that

$n_{d}=\frac{f}{\sqrt{1-2 \varphi / M^{2}}}$.

Only the dust density contains a Mach number $M=U / C_{d a}$ (coming from inertial effects on its flow) and it is the sole supersonic species.

The basic set of equations is closed by Poisson's equation, which gives after integration an energy-like integral,

$$
\frac{1}{2}\left(\frac{d \varphi}{d x}\right)^{2}+S(\varphi)=0
$$

Here $S(\varphi)$ is the Sagdeev pseudopotential,

$$
\begin{aligned}
S(\varphi):= & \frac{1-f}{\tau}(1-\exp [-\tau \varphi])+f M^{2}\left(1-\sqrt{1-\frac{2 \varphi}{M^{2}}}\right) \\
& +\frac{1+15 \alpha}{1+3 \alpha}-\frac{1+15 \alpha-12 \alpha \varphi+4 \alpha \varphi^{2}}{1+3 \alpha} \exp [\varphi] .
\end{aligned}
$$

This can be analyzed as in classical mechanics, in terms of the properties of $S(\varphi)$. By construction and assumption we see that $S(0)=S^{\prime}(0)=0$, where primes refer to derivatives with respect to $\varphi$. We furthermore require $S^{\prime \prime}(0)<0$, causing the origin to be a (local) unstable maximum and ensuring that the nonlinear structures are superacoustic in a global sense. At the same time, this yields the minimal $M$ for their existence,

$M^{2}>M_{s}^{2}:=\frac{f(1+3 \alpha)}{1-\alpha+\tau(1-f)(1+3 \alpha)}$.
However, in order to have a solution, we need to encounter roots of $S(\varphi)$ outside $\varphi=0$. Single roots give hill- or dip-like solitons, whereas for double roots $\varphi$ changes from one value at $-\infty$ to another at $+\infty$ and we have potential kinks. In the following section we analyze the existence domains for nonlinear solitons and kinks in a systematic way, rather than focus on limited $M$ values and compositional parameters, for which the numerics give solutions.

\section{Positive and negative solitons}

Both the nonthermal electron and Boltzmann ion densities are well behaved for all $\varphi$, whereas the cold dust undergoes an infinite compression for $\varphi \rightarrow M^{2} / 2$, which restricts the range $\varphi>0$ for which $S(\varphi)$ is real. Equation (2) indicates that solutions with $\varphi>0$ entail a rarefaction in the ions, whereas (1) and (3) likewise give a compression in the electrons and the dust, respectively. Indeed, for $\alpha<1$ the expression for $n_{e}$ is monotonically increasing with $\varphi$. The existence of such solutions requires that a root of $S(\varphi)$ is encountered before $\varphi=M^{2} / 2$, and hence needs $S\left(M^{2} / 2\right)>0$. Upper limiting $M$ numbers follow from $S\left(M^{2} / 2\right)=0$ and together with the lower $M_{S}$ bound, indicate that positive structures exist for $M$ inside a range, at given $f$.

The top diagram in Fig. 1 shows that if one picks $f$ and starts with $M$ just above a lower curve and ends just below the corresponding upper curve, all intermediate $M$ generate positive solitons, which get stronger at larger $M$. For completeness, the curves go down to $f=0$ and up to $f=1$, but these limits do not correspond to realistic physics, as for $f=0$ there is no dust left, hence no inertia, whereas for $f=1$ there are no ions left.

The top diagram in Fig. 1 also shows that the upper curves are not very sensitive to changes in $\alpha$, at fixed $\tau$, but the lower ones are, so that for $\alpha<0.285$ the corresponding curves do not intersect for $0<f<1$. However, when the nonthermal distribution includes too many superthermal electrons $(\alpha>0.285)$, the sluggish dust can not sustain the solitons alone, and some more mobile ions are needed for that.

For $\varphi<0$ all densities, as well as $S(\varphi)$, are well behaved and there are no immediate restrictions on $\varphi$. Possible negative solitons are characterized by a compression in the ions and a rarefaction in the electrons and the dust. Combining $\lim _{\varphi \rightarrow-\infty} S(\varphi) \rightarrow-\infty$ with $S^{\prime \prime}(0)<0$ means that negative roots, if they exist, should come in pairs. Hence, a range of possible solitons ends when a kink is encountered, giving the natural upper limits on $M$ and on the negative soliton amplitudes (Baboolal et al., 1990). The double root conditions have been considered for given $\alpha, f$ and $\tau$, from which it transpires that there is only a limited existence range in $f$, near the higher dust density end, as illustrated in the bottom diagram of Fig. 1. For $\alpha=0.2$ negative kinks are only possible between $0.95<f \leq 1$, and completely impossible for $\alpha<0.148$. The range of the curved wedge-shaped domains 

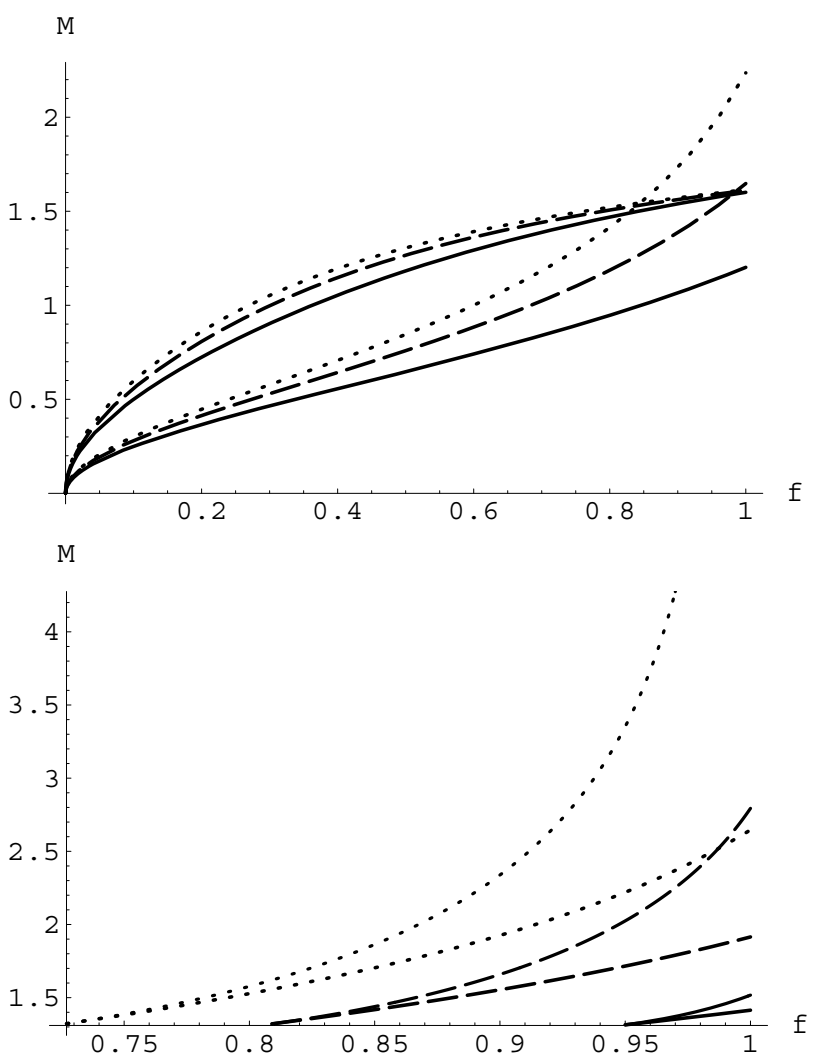

Fig. 1. Existence curves for positive solitons (top diagram), for $\alpha=0.1(-), \alpha=0.3(--)$ and $\alpha=0.5(\cdots)$, and for negative solitons (bottom diagram), for $\alpha=0.2(-), \alpha=0.4(--)$ and $\alpha=0.6(\cdots)$, all at $\tau=1$. At each $\alpha$ the lower curves express the soliton condition, the upper ones that positive roots are limited by infinite dust compression (top diagram), negative ones by the occurrence of kinks (bottom diagram).

gets wider at larger $\alpha$. The existence of these negative kinks needs sufficient electron nonthermality and sufficient positive dust. Theoretically, the ion presence is not even required.

Combining both diagrams of Fig. 1, there thus are three regions, as shown in Fig. 2.

First, there is a large region in parameter space where only positive solitons can occur, which extends all the way towards $f=0$. Beyond the intersection of the two upper curves (e.g. near $f=0.94$ for $\alpha=0.3$ and $\tau=1$ ) there is a region where only negative solitons can be generated, at fairly large $M$ values. Finally, below both upper curves and above the soliton condition there is region where both types of solitons coexist.

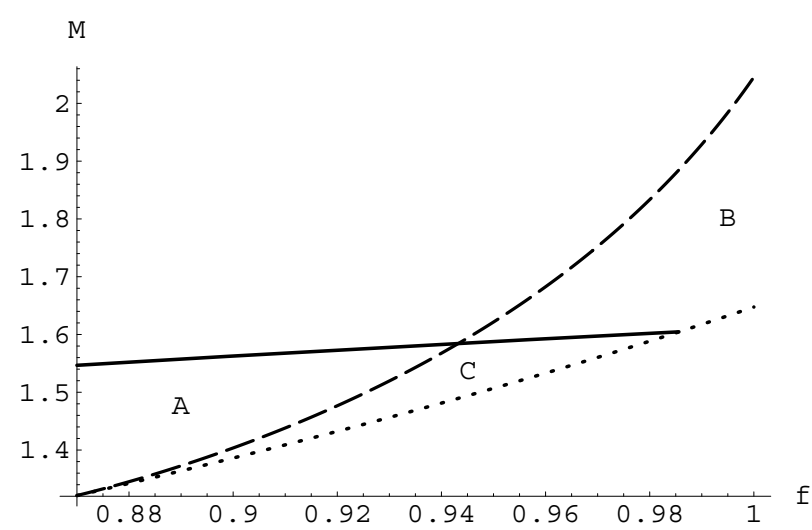

Fig. 2. Existence curves for positive and negative solitary waves, for values of $\alpha=0.3$ and $\tau=1$. The lowest curve is the solitary wave condition $(\cdots)$, the upper ones are the limits on positive $(-)$ and negative $(--)$ potential solutions. In region $A$ there are only positive solitary waves, in region $B$ only negative solitary waves and in region $C$ both types of solutions coexist. Region $B$ ends at $f=1$, whereas most of the region $A$ (between $f=0$ and $f=0.87$ ) has not been shown for reasons of graphical clarity.

\section{Further remarks and summary}

To see the influence of the ion temperature, we choose $\alpha$ and let $\tau$ vary. Positive solitons exist for all $0<f<1$, at small enough $\alpha$, and the boundaries of their existence domains are but little influenced by the ion temperature, as seen in the top diagram of Fig. 3.

Negative solitons are limited by a potential kink, conditions which are much more sensitive to parameter changes. In the bottom diagram of Fig. 3, existence domains (for $\alpha=0.3$ ) start for $\tau=0.2$ at $f=0.60$, for $\tau=0.6$ at $f=0.79$, for $\tau=1$ at $f=0.87$ and for $\tau=5$ at $f=0.95$. Since we have used $T_{e}$ for the temperature normalization, increasing $\tau=T_{e} / T_{i}$ means decreasing $T_{i}$.

For the converse plasma model comprising Boltzmann electrons, nonthermal positive ions and cold positive dust, we have been unable to find negative roots, within the limits of numerical accuracy over wide parameter ranges. There are only positive solutions, the character of which is fully determined by the sign of the supersonic, sluggish dust. With the appropriate changes from negatively to positively charged dust, this corroborates earlier conclusions (Verheest and Pillay, 2008) for negative dust, nonthermal electrons and Boltzmann positive ions.

Summing up, it is the cold dust which gives rise to solitons of the same sign as the dust: positive here, negative in our earlier paper (Verheest and Pillay, 2008), with no obvious limits on $f$ in $[0,1]$. To obtain solitary structures of the other polarity, a sufficient degree of nonthermality is needed in the Cairns distribution of the hot species having a sign opposite to that of the cold dust, viz. electrons here, (positive) ions in our earlier paper. These solitons and double layers 


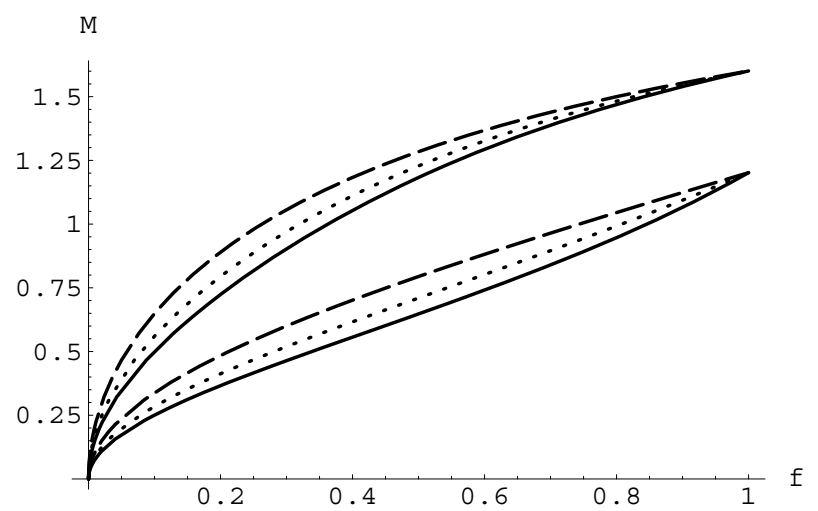

M

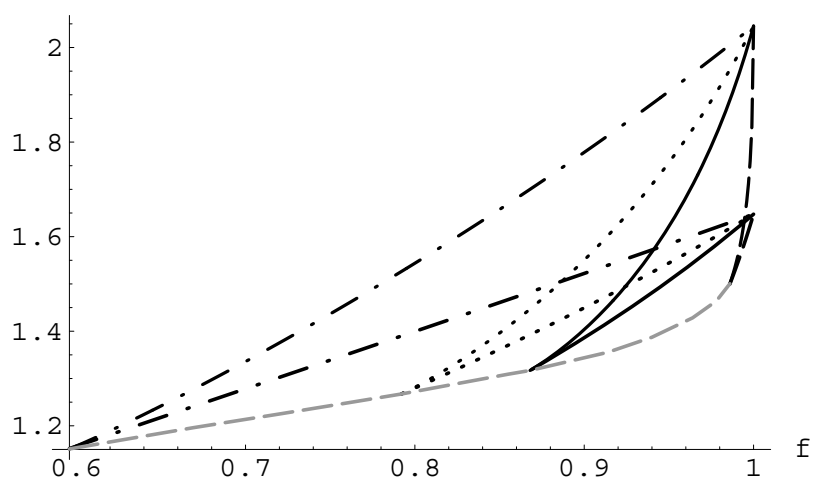

Fig. 3. In the top diagram, existence domains for positive solitons are given at fixed $\alpha=0.1$ but $\tau=0.2(--), \tau=0.6(\cdots)$ and $\tau=1(-$ ). The bottom diagram shows the existence domains for negative solitons at $\alpha=0.3$ but $\tau=0.2(-\cdots), \tau=0.6(\cdots), \tau=1(-)$ and $\tau=5(--)$. The dashed grey line connects the starting points for the different existence domains as function of increasing $\tau$.

occur in a restricted range for $f$ near the $f=1$ limit. Hot species, even if nonthermally distributed, of the same sign as the cold dust cannot generate solitary structures with an opposite polarity and hence have been treated as Boltzmann distributed, in order not to overburden our mostly analytical analysis. Only the upper curves limiting $M$ at a given $f$ for the double layers have been generated numerically.

To conclude, we have studied large dust-acoustic structures in a model with nonthermal electrons, Boltzmann ions and cold positive dust. Positive solitons can easily be generated and are limited by infinite dust compression. Negative structures, possible only for $\alpha>0.148$, need enough electron nonthermality and are limited by the occurrence of negative potential kinks. Our results might help understand nonlinear structures in dusty plasmas containing nonthermal electrons, as in certain heliospheric environments.
Acknowledgements. FV thanks the FWO (Vlaanderen) for a research grant.

Edited by: P.-L. Sulem

Reviewed by: I. Kourakis and another anonymous referee

\section{References}

Asbridge, J. R., Bame, S. J., and Strong, I. B.: Outward flow of protons from Earths bow shock, J. Geophys. Res., 73, 5777-5782, 1968.

Baboolal, S., Bharuthram, R., and Hellberg, M. A.: Cut-off conditions and existence domains for large-amplitude ion-acoustic solitons and double layers in fluid plasmas, J. Plasma Phys., 44, 1-23, 1990.

Baluku, T. K., Hellberg, M. A., and Mace, R. L.: Solitons in dusty plasmas with positive dust grains, Phys. Plasmas, 15, 033701, 2008.

Barkan, A., Merlino, R. L., and D'Angelo, N.: Laboratory observation of the dust-acoustic wave mode, Phys. Plasmas, 2, 35633565, 1995.

Cairns, R. A., Mamun, A. A., Bingham, R., Boström, R., Dendy, R. O., Nairn, C. M. C., and Shukla, P. K.: Electrostatic solitary structures in non-thermal plasmas, Geophys. Res. Lett., 22, 2709-2712, 1995.

Feldman, W. C., Anderson, R. C., Bame, S. J., Gary, S. P., Gosling, J. T., McComas, D. J., Thomsen,M. F., Paschmann, G., and Hoppe, M. M.: Electron velocity distributions near the Earths bow shock, J. Geophys. Res., 88, 96-110, 1983.

Futaana, Y., Machida, S., Saito, Y., Matsuoka, A., and Hayakawa, H.: Moon-related nonthermal ions observed by Nozomi: Species, sources, and generation mechanisms, J. Geophys. Res., 108, 1025, doi:10.1029/2002JA009366, 2003.

Lundin, R., Zakharov, A., Pellinen, R., Borg, H., Hultqvist, B., Pissarenko, N., Dubinin, E. M., Barabash, S. W., Liede, I., and Koskinen, H.: 1st measurements of the ionospheric plasma escape from Mars, Nature, 341, 609-612, 1989.

Maharaj, S. K., Pillay, S. R., Bharuthram, R., Reddy, R. V., Singh, S. V., and Lakhina, G. S.: Arbitrary amplitude dust-acoustic double layers in a non-thermal plasma, J. Plasma Phys., 72, 43-58, 2006.

Mamun, A. A.: Coexistence of positive and negative solitary potential structures in dusty plasma, Phys. Lett. A, 372, 686-689, 2008.

Mamun, A. A. and Shukla, P. K.: Solitary potentials in cometary dusty plasmas, Geophys. Res. Lett., 29, 1870, doi:10.1029/2002GL015219, 2002.

McKenzie, J. F.: The fluid-dynamic paradigm of the dust-acoustic soliton, J. Plasma Phys., 67, 353-362, 2002.

Mendoza-Briceño, C. A., Russell, S. M., and Mamun, A. A.: Large amplitude electrostatic solitary structures in a hot non-thermal dusty plasma, Planet. Space Sci., 48, 599-608, 2000.

Rao, N. N., Shukla, P. K., and Yu, M. Y.: Dust-acoustic waves in dusty plasmas, Planet. Space Sci., 38, 543-546, 1990.

Sakanaka, P. H. and Shukla, P. K.: Large amplitude solitons and double layers in multicomponent dusty plasmas, Physica Scripta, T84, 181-183, 2000.

Shukla, P. K. and Mamun, A. A.: Introduction to Dusty Plasma Physics, IOP Press, 2002. 
F. Verheest and S. R. Pillay: Plasma solitons with nonthermal electrons and positive dust

Verheest, F.: Nonlinear dust-acoustic waves in multispecies dusty plasmas, Planet. Space Sci., 40, 1-6, 1992.

Verheest, F.: Waves in Dusty Space Plasmas, Kluwer, 2000.
Verheest, F., Cattaert, T., Lakhina, G. S., and Singh, S. V.: Gasdynamic description of electrostatic solitons, J. Plasma Phys., 70, 237-250, 2004.

Verheest, F. and Pillay, S. R.: Large amplitude dust-acoustic solitary waves and double layers in nonthermal plasmas, Phys. Plasmas, 15, 013703, 2008. 